Gynäk. Rdsch. 1975;15(Suppl. 1):1-2

\title{
Contents, Vol. 15, Supplement 1, 1975
}

1. Hauptthema Die Friihgeburt

ler sujet principal La prématurité

Muralt, G. de (Berne): Introduction

Papiernik, E. et Schneider, L. (Clamart): Etiologie de la prématurité

Hinselmann, M. et De Grandi, P. (Bale): Le diagnostic prenatal de la prématurité ...

Béguin, F. (Geneve): La prophylaxie de Taccouchement premature

18

Relier, J.P. (Paris): Particularités pathophysiologiques et cliniques du premature ....

Due, G. (Zurich): Mesures preventives de la mortalite neonatale et de la morbidite cérébrale associées à la prématurité 34

Mitteilungen zum I. Hauptthema

Communicatons se rapportant au ler sujet principal

Brunner, J.; Keller, P.J.; Schmid, J. und Kunz, J. (Zurich): Gesamtphospholipide und Lezithin-Sphingomyelin-Ratio zur Bestimmung der fetalen Lungenreife 39

Arendt, J. et Béguin, F. (Geneve): Acide 5-hydroxy-indole-acétique: reflet du système nerveux central foetal?

Sidiropoulos, D. und Muralt, G. von (Bern): Postnatale Lungenreifebestimmung .... 47 Buess, H; Hinselmann, M.; Stahel, Th.; Harms, K; Roemer, M. und Ramzin, M.

(Basel): Sectio und Friihgeburt 52

Brun del Re, R.; De Grandi, P.; Buess, H; Hinselmann, M. und Ramzin, M. (Basel):

Beziehung zwischen Latenzzeit des Blasensprungs und hyalinen Membranen bei

Friihgeburt 55

Richter, R.; Hammacher, K. und Hinselmann, M. (Basel): Die Prophylaxe der drohenden Friihgeburt. Klinische Ergebnisse einer prospektiven Vergleichsstudie mit

Ritodrine und Buphenin 58

Hofer, U. (Münsterlingen): Langzeitapplikation von Buphenin-hchl (Dilydrin retard).

Resultate unter besonderer Berücksichtigung des Glukosestoffwechsels 60

Baur, B.; Hinselmann, M.; Ramzin, M. und Jann, F.X. Bern/Basel): Wertigkeit von normalen und abnormen Massen des biparietalen Durchmessers zur Voraussage des Kindsgewichtes 63

Index

2

2. Hauptthema Gynäkologische Urologie

2e sujet principal Urologie gynécologique

Rütte, B. von (Bern): Urge-und Dranginkontinenz

Haag,B. (Basel): Detrusorstörungen und instabile Blase

Hochuli, E. (Münsterlingen): Operative Behandlung der schweren Urininkontinenz zweiten Grades 79

Dreher, E. (Bern): Ergebnisse nach Inkontinenzoperationen 80 
Schmid, J. und Baertschi, U. (Zurich): Postoperative urologische Komplikationen ...

Held, E. (Zurich): Urethro-Vaginal-Fisteln mit Harninkontinenz

96

Zingg, E.J. (Bern): Harnfisteln

99

Mitteilungen zum 2. Hauptthema Communications se rapportant au 2e sujet principal Moser, R.; Sebeseri, O. und Poretti, G. (Bern): Die Clipsmarkierung von Uterus und Harnblase zur Dosimetrie fur die intrakavitäre und perkutane Strahlentherapie . 105 Bodingbauer, J.; Eberhard, K. und Hochuli, E. (Münsterlingen): Die Puborektalisplastik nach Franz, eine Alternative zu den kombinierten Schlingenoperationen 108 Bührig, H; Schmid, J. und Soyka, E. (Zurich): Langzeitergebnisse nach Operationen der reinen Stressinkontinenz 113

Eduah, S.B. und Dreher, E. (Bern): Die Wirkung des Parasympathikomimetikums, Distigmin-Bromid (Ubretid ${ }^{\circledR}$ ) auf den Urethralverschluss bei der Frau 115 Herrmann, U. (Biel): Bakteriurie in graviditate 118

Herrmann, U. (Biel): Bakteriurie bei gynäkologischen Erkrankungen

Kunz, J. und Baertschi, U. (Zurich): 1st eine postoperative Chemoprophylaxe des Harnweginfektes sinnvoll? 125

Litschgi, M.S.; Benz, J.J. und Glatthaar, E. (Winterthur): Blasenstein als Komplikation nach gynäkologischen Operationen 127

Schweizerische Gesellschaft für Gynäkologie

Bericht über die Jahresversammlung, 19.-21. Juni 1975 in Nyon

Société Suisse de Gynécologie

Compte rendu de l'Assemblée annuelle, 19-21 juin 1975 àNyon Redaktor - Rédacteur: H. Erb, Liestal 